\title{
Accurate Circuit Modeling of Fishnet Structures for Negative-Index-Medium Applications
}

\author{
Víctor Torres, Francisco Mesa, Fellow, IEEE, Miguel Navarro-Cía, Senior Member, IEEE, \\ Raúl Rodríguez-Berral, Miguel Beruete, and Francisco Medina, Fellow, IEEE
}

\begin{abstract}
Metallic plates with a two-dimensional (2D) periodic distribution of sub-wavelength apertures are known to exhibit extraordinary transmission of electromagnetic waves. Stacking two or more of such plates gives place to the so-called fishnet structures, which constitute a popular way of achieving an effective negative index medium at frequencies ranging from microwaves to optics. Unfortunately, a general wideband equivalent circuit has not yet been proposed to facilitate its understanding and design. This work presents this circuit model with closed-form expressions for the circuit elements, thus making it possible to obtain the electrical response for this class of structures in a very efficient way. This procedure is much faster than alternative numerical methods at the same time that it retains a high level of accuracy when compared with some other oversimplified models. The circuit model also provides a simple rationale as well as a good physical insight in order to explain the qualitative behavior of such structures, independently of the number of stacked layers.
\end{abstract}

Index Terms-Equivalent circuit models, extraordinary transmission (ET), fishnet structures, metamaterials, negative index materials (NIMs).

\section{INTRODUCTION}

W ITHIN THE realm of metamaterials, major efforts have been focused on realizing structures with effective negative index of refraction, the so-called negative index metamaterials (NIMs). Apart from the pure scientific interest on their peculiar electromagnetic response, the study of these media has been triggered by many potential practical applications including superlensing, sensing and other novel

Manuscript received March 12, 2015; revised August 13, 2015; accepted November 17, 2015. This work was supported by the Spanish Ministry of Economy and Competitiveness with European Union Feder Funds (under grants TEC2011-28664-C02-01, TEC2014-51902-C2-2-R, TEC2013-41913-P, and Consolider EMET CSD2008-00066, and by the Spanish Junta de Andalucía under Project P12-TIC-1435). The work of M. Navarro-Cía was supported by the Imperial College Junior Research Fellowship and the Birmingham Fellowship. The work of M. Beruete was supported by the Spanish Government via RYC-2011-08221.

V. Torres and M. Beruete are with the Antenna Group-TERALAB, Universidad Pública de Navarra, Pamplona, 31006, Spain (e-mail: victor.torres@unavarra.es; miguel.beruete@unavarra.es).

M. Navarro-Cía was with the Imperial College London, London SW7 2AZ, U.K. He is now with the School of Physics and Astronomy, University of Birmingham, Birmingham, B15 2TT, U.K. (e-mail: M.Navarro-Cia@bham.ac.uk).

R. Rodríguez-Berral and F. Mesa are with the Department of Applied Physics 1, ETSII, 41012, Seville, Spain (e-mail: rrberral@us.es; mesa@us.es).

F. Medina is with the Department of Electronics and Electromagnetism, Faculty of Physics, University of Sevilla, 41012, Seville, Spain (e-mail: medina@us.es).

Color versions of one or more of the figures in this paper are available online at http://ieeexplore.ieee.org.

Digital Object Identifier 10.1109/TMTT.2015.2504441 electromagnetic devices [1], [2]. The first experimental implementation of a NIM was done at microwave frequencies by combining thin metallic wires and split-ring resonators [3] but the achievement of NIMs at higher frequencies (infrared or optics) is not trivial because not all topologies retain the required strong magnetic response in such spectral windows [4]. In this regard, the fishnet structure has outperformed other topologies [5] and can be applied over a very wide range of frequencies. The fishnet is a multi-layered structure that comprises two (or more) metallic periodic hole arrays separated by dielectric slabs. Roughly speaking, the negative index of refraction in the fishnet stems from the virtual current loop formed by the coupling between the layers and the surface currents rounding the holes provided the perforated metal screens are operating in the extraordinary transmission (ET) [6], [7] regime. Its simple geometry facilitates the fabrication at the nanoscale, making it one of the most attractive metamaterials so far. Hence, the fishnet is currently the preferred option for synthesizing NIMs from microwaves [8]-[10] to the optical regime [5], [11], [12], and has been successfully employed for designing quasi-optical devices such as polarizers, lenses, and demultiplexers [13]-[17].

In the context of electromagnetic periodic structures, analyses based on equivalent circuits (ECs) have a long tradition [18]-[26]. The main advantage of the circuit theory approach is that qualitative as well as quantitative predictions are feasible without performing intensive full-wave numerical simulations. Therefore, an EC for the fishnet metamaterial is also highly desired. In the study of the ET phenomenon, transmission line concepts and ECs have been able to provide successful explanations to ET through sub-wavelength hole arrays [27]. The electrically small apertures are seen as reactive discontinuities modeled with lumped elements (or, sometimes, distributed elements) in the path of an electromagnetic mode propagating along an artificial waveguide. This approach reduces the original problem to a classical waveguide discontinuity problem [28]. Further works have extended this technique to similar ET metamaterials in order to analyze the importance of the aperture type [29]-[31], polarization [32], [33], metal losses [34], the inclusion of dielectric slabs [24], [35] or the design of polarizing devices [36].

Likewise, the fishnet has also been analyzed from an EC point of view in order to obtain a more intuitive explanation than alternative interpretations based on diffraction orders of complex waves, Floquet-Bloch modes or surface plasmons [37]-[39], while retaining the underlying physics of the problem and the accuracy of the results. The response of the basic fishnet structure can be understood in terms of an elementary backward transmission line having series capacitance and shunt inductance. The series capacitance comes from the electric coupling between consecutive layers and the shunt inductance represents 
the inductive nature of the sub-wavelength apertures [40]. Several other EC models can be found in the literature dealing with problems involving stacked perforated metal layers [41]-[46]. However, most of these works are of heuristic nature and do not provide closed-form expressions for the circuit elements [43], [44] or their scope is limited to certain configurations and structural parameters (small unit cells, large longitudinal period and so on). Thus, a general formulation valid for structures having unit cells of arbitrary electrical size separated by arbitrary distances would be of great interest from a practical point of view. Computationally intensive brute force calculations would then be avoided at the same time that a better physical insight could be gained.

In this work, a simple analytical circuit model that uses lumped elements and transmission lines is derived to study the transmission of electromagnetic waves through a fishnet structure with a finite number of layers (see Fig. 1). The structure is assumed periodic and infinite along the $x$ and $y$ transverse directions. The incident plane wave is impinging obliquely to the structure and its electric field is assumed to be directed along the $y$ direction (the wave will be considered of TM nature at normal incidence). The attention is mainly focused on the separation between layers because it tunes the effective refractive index of the fundamental band [40]. Therefore, two different operation regimes are studied: large and short separation, which respectively produce weak and strong coupling between layers through higher-order modes. Note that interaction through the fundamental mode is always present for any separation as this mode has not cutoff frequency. For each regime, we investigate the configurations with air and a dielectric material with permittivity $\varepsilon_{r}=1.4$ as inter-spacing layers. Different closed-form expressions for the circuit elements are presented for all situations. The physical insight provided by the EC is corroborated with the study of the electromagnetic fields at the most relevant frequencies. Although our study is focused on microwaves to simplify the model for metals to perfect electric conductor, the method could be extended to consider other models such as Drude or finite conductivity models [47], [48]. This paper will be organized as follows. Section II will show our proposed model for fishnet structures with a finite number of layers. Section III will present comparison results of the transmission coefficient obtained with the circuit model and with full-wave simulations under normal and oblique incidence for different longitudinal periods. Also, the study of the electromagnetic fields at the most relevant frequencies. Finally, some concluding remarks will be summarized in Section IV.

\section{CiRCUit MODELING}

The use of ECs to model many propagation/scattering problems is a common and very fruitful practice. However, most of the analyses just propose (in a heuristic way) the topology of an equivalent network that has a behavior very similar to the original problem over a certain frequency band. The values of the parameters of the equivalent circuit are then obtained from some full-wave simulations of the complete electromagnetic problem (these simulations often need to be carried out at particular frequency points that are only known a posteriori). Although this strategy is useful in many situations, our aim here is to derive the appropriate circuit topology in a rigorous way from first principles and to give the values of the circuit parameters in closed form. This standpoint has been used in the past by some of the authors of the present work to model other periodic structures

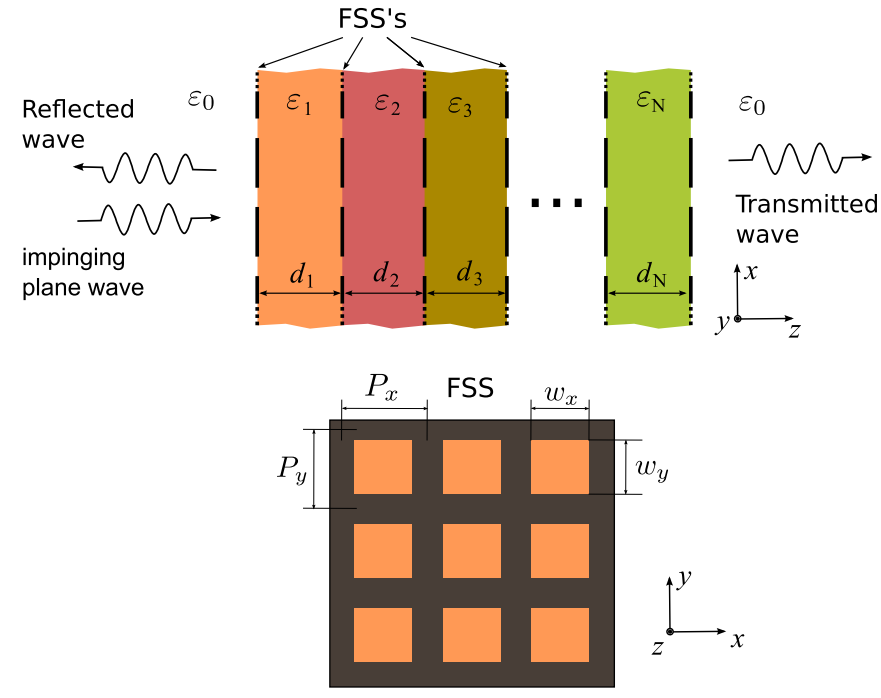

Fig. 1. Schematic representation of a fishnet structure with a finite number of layers. The upper panel shows a longitudinal cross section and the bottom panel a front view of the structure.

[35], [49]-[51], and it is now extended to stacked 2D geometries so that the problem under study (multilayer fishnets) can be covered.

In [51] it was shown that the key aspect that makes it possible to easily obtain an equivalent network for the problem of $N$ stacked metallic slit gratings (1D version of the problem of interest in that paper) comes from the decomposition of the two-coupled metallic screens problem into "internal" and "external" sub-problems as a consequence of the Equivalence Theorem [52]. The extension of this idea to a pair of coupled aperture-type frequency selective surfaces (FSS's) (the most basic form of fishnet structure) is immediate as long as the FSS's are of slot-type nature too (the procedure is not directly applicable to patch-based FSS's). Thus, following [51], the problem of the two-coupled aperture-type FSS's shown in Fig. 2(a) has the equivalent $\pi$ network shown in Fig. 2(b), or the internal/external version given in Fig. 2(c). It is now straightforward to realize that the equivalent network of, say, four cascaded identical FSS's such as that shown in Fig. 3(a) is the one given in Fig. 3(b).

Our attention now focuses on the calculation of the few admittances involved in the analysis of the coupled aperture-based FSS. For that purpose, the standard application of the even/odd excitation approach [52] to the problem in Fig. 2(a) makes it possible to reduce our original problem to the analysis of problems with just one single FSS and electric/magnetic walls at half the distance between the original FSS's, as shown in Fig. 4. The analysis of this much simpler structure will follow some of the guidelines reported in [35], [50]. When this analysis is carried out, the final results for the equivalent admittance corresponding to the incident TM plane wave (electric field directed along the $y$ direction) are the following (see Fig. 4):

$$
\begin{aligned}
Y_{\mathrm{eq}}^{(\mathrm{e} / \mathrm{o})}=\sum_{n, m}^{\infty} A_{n m}^{\mathrm{TM}}\left(Y_{n m}^{\mathrm{TM}(0)}\right. & \left.+Y_{n m, \mathrm{e} / \mathrm{o}}^{\mathrm{TM}(\mathrm{in})}\right) \\
& +A_{n m}^{\mathrm{TE}}\left(Y_{n m}^{\mathrm{TE}(0)}+Y_{n m, \mathrm{e} / \mathrm{o}}^{\mathrm{TE}(\mathrm{in})}\right)
\end{aligned}
$$

where the prime in the summation means that it excludes the fundamental $\mathrm{TM}_{00}$ harmonic associated with the impinging, 


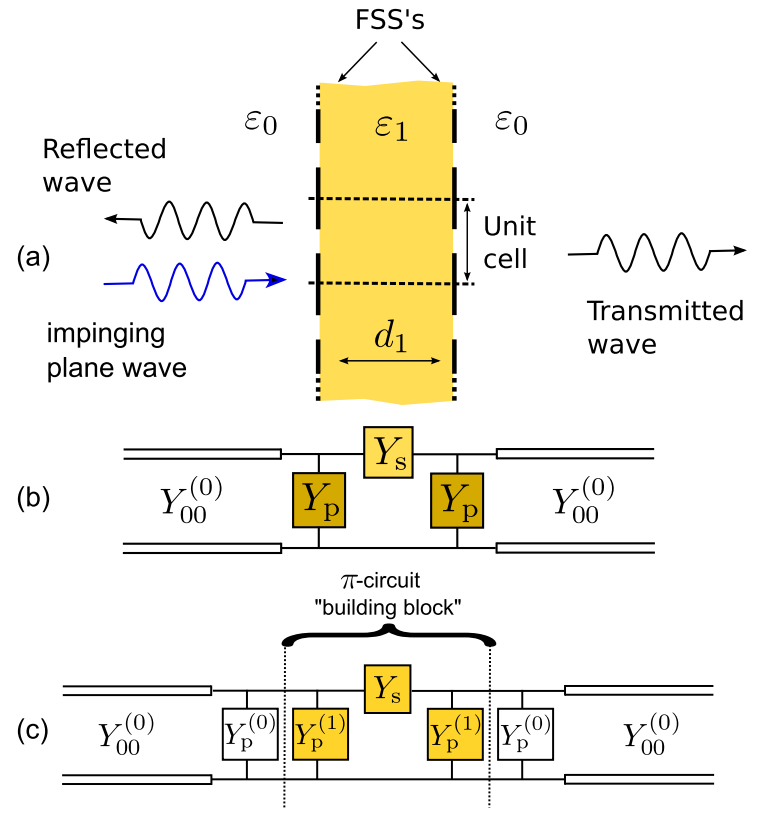

Fig. 2. (a) Two coupled apertured-based FSS's. (b) Equivalent circuit for the scattering of the impinging plane wave in the two-coupled FSS structure. The coupled screens are represented by a $\pi$-network. (c) Equivalent circuit already shown in (b) but now showing the decomposition of the parallel element into an external admittance $Y_{\mathrm{p}}^{(0)}$ and an internal admittance $Y_{\mathrm{p}}^{(1)}$.

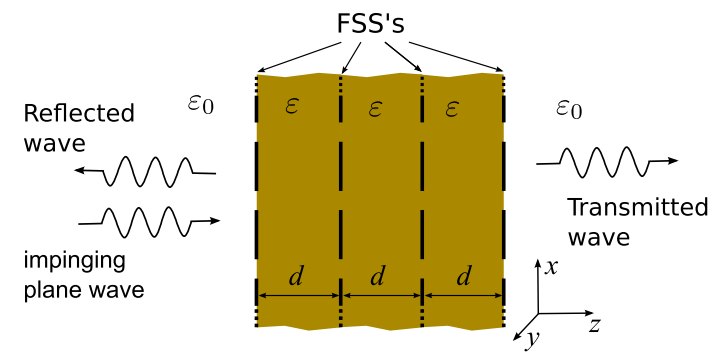

(a)

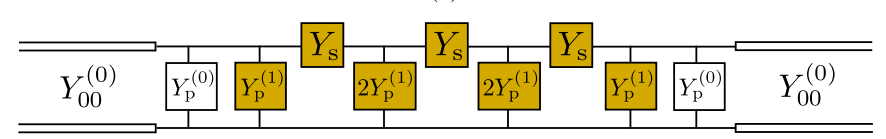

(b)

Fig. 3. (a) Stack of four coupled apertured-based FSS's. (b) Equivalent circuit for the scattering of the impinging plane wave in the stacked structure.

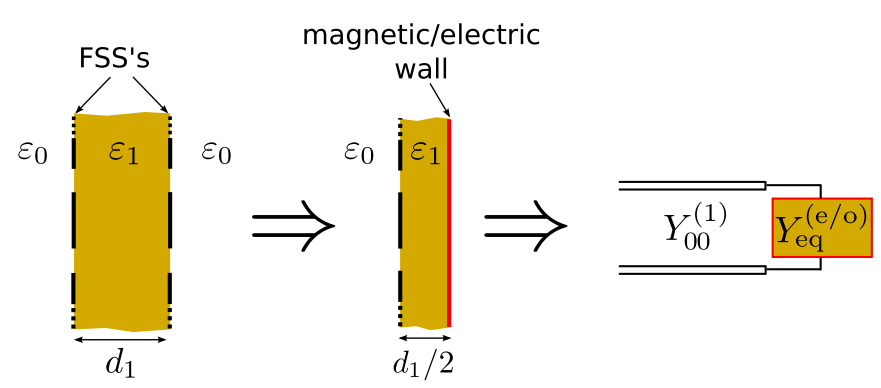

Fig. 4. Pair of coupled FSS's and its associated even/odd excitation (magnetic/ electric wall) half-problems with their equivalent circuits.

reflected, and transmitted uniform plane waves (the $\mathrm{TE}_{00}$ harmonic is also excluded since it cannot exist in the present configuration). The superscripts "e/o" stand for "even/odd" excitation and "TM/TE" refers to the nature of the considered harmonic.
The admittances appearing in (1) are given by the following analytical expressions:

$$
\begin{aligned}
& Y_{n m, \mathrm{e} / \mathrm{o}}^{\mathrm{TE} / \mathrm{Tin})}=\mathrm{j} Y_{n m}^{\mathrm{TE} / \mathrm{TM}(1)} \begin{cases}\tan \left(\frac{\beta_{n m}^{(1)} d_{1}}{2}\right) & \text { even excit. } \\
-\cot \left(\frac{\beta_{n m}^{(1)} d_{1}}{2}\right) & \text { odd excit. }\end{cases} \\
& Y_{n m}^{\mathrm{TM}(i)}=\frac{\omega \varepsilon_{i}}{\beta_{n m}^{(i)}} \quad Y_{n m}^{\mathrm{TE}(i)}=\frac{\beta_{n m}^{(i)}}{\omega \mu_{0}} \\
& \beta_{n m}^{(i)}=\sqrt{\varepsilon_{r, i} k_{0}^{2}-k_{x n}^{2}-k_{y m}^{2}} \quad(i=0,1) \\
& k_{x n}=k_{x 0}+\frac{2 \pi n}{P_{x}} \quad k_{y m}=k_{y 0}+\frac{2 \pi m}{P_{y}}
\end{aligned}
$$

with $k_{0}$ being the vacuum wavenumber, $\omega$ the angular frequency,

$$
\mathbf{k}_{t}=\left(k_{x 0}, k_{y 0}\right)=-\left(k_{0} \sin \theta \cos \varphi, k_{0} \sin \theta \sin \varphi\right)
$$

the wavevector of the impinging plane wave, and index "(0)" refers to the external free-space region while index "(1)" refers to the internal region between metal screens. The angles $\theta$ and $\varphi$ define the direction ofthe impinging wave as usual. However, in this paper, it will only be considered incidence along principal planes; i.e., $\varphi$ will be allowed to take only the values $0^{\circ}$ and $90^{\circ}$. The general case of conical incidence could be treated using the method reported in [50]. It is worth mentioning that the given expression for $Y_{\mathrm{eq}}^{(\mathrm{e} / \mathrm{o})}$ accounts for all the high-order harmonics excited at the discontinuity. This means that the possible effects associated with high-order modes caused by the close proximity between screens are properly incorporated into the model.

Assuming that the tangential electric field at the aperture is directed along $y\left(\varphi=90^{\circ}\right.$ for TM incidence or $\varphi=0^{\circ}$ for TE incidence), the $A_{n m}^{\mathrm{TE} / \mathrm{TM}}$ coefficients in (1) are given by

$$
\begin{aligned}
& A_{n m}^{\mathrm{TM}}=\frac{k_{y m}^{2}}{k_{x n}^{2}+k_{y m}^{2}}\left|\frac{\widetilde{E}_{a}\left(k_{x n}, k_{y m}\right)}{\widetilde{E}_{a}(0,0)}\right|^{2} \\
& A_{n m}^{\mathrm{TE}}=\frac{k_{x n}^{2}}{k_{x n}^{2}+k_{y m}^{2}}\left|\frac{\widetilde{E}_{a}\left(k_{x n}, k_{y m}\right)}{\widetilde{E}_{a}(0,0)}\right|^{2}
\end{aligned}
$$

with $\widetilde{E}_{a}\left(k_{x n}, k_{y m}\right)$ being the Fourier transform of the spatial profile of the tangential electric field in the aperture. This spatial profile is not known a priori but can be very well-approximated for many practical cases. In particular, for rectangular apertures and for the type of illumination considered in this paper, we have employed the spatial profile suggested in [53] (see the Appendix). Here, it is important to point out that the above approximation turns out to be quite accurate up to frequencies close to the second self-resonance of the individual aperture that is compatible with the impinging wave [50]. In practice, this means that the aperture has not to be sub-wavelength for the circuit approach to be applied with reliability. Actually, for normal incidence, the size of the apertures can be even greater than a wavelength [50]. It is also noteworthy that $A_{0 m}^{\mathrm{TE}}=0 \forall m$ and also that $A_{n 0}^{\mathrm{TM}}=0 \forall n \neq 0$. This is consistent with the fact that $\mathrm{TE}_{0 m}$ and $\mathrm{TM}_{n 0}$ harmonics cannot be excited due to the symmetries of the unit cell and the polarization of the incident plane wave.

Our next step is to find the relationship between the defined $Y_{\mathrm{eq}}^{(\mathrm{e} / \mathrm{o})}$ and the admittances of the equivalent $\pi$-network for a 
pair of coupled perforated screens. Since the even and odd excitations correspond respectively to open and short-circuit terminations at the middle plane of the equivalent circuit, the parallel and series admittances of the $\pi$-network in Fig. 2 are found to be

$$
Y_{\mathrm{p}}=Y_{\mathrm{eq}}^{(\mathrm{e})}, \quad Y_{\mathrm{s}}=\frac{1}{2}\left[Y_{\mathrm{eq}}^{(\mathrm{o})}-Y_{\mathrm{eq}}^{(\mathrm{e})}\right] .
$$

From these expressions, it is straightforward to identify the admittances appearing in Fig. 3(b) to obtain

$$
Y_{\mathrm{p}}=Y_{\mathrm{p}}^{(0)}+Y_{\mathrm{p}}^{(1)}
$$

where

$$
\begin{aligned}
Y_{\mathrm{p}}^{(0)} & =\sum_{n, m}^{\infty} A_{n m}^{\mathrm{TM}} Y_{n m}^{\mathrm{TM}(0)}+A_{n m}^{\mathrm{TE}} Y_{n m}^{\mathrm{TE}(0)} \\
Y_{\mathrm{p}}^{(1)} & =\mathrm{j} \sum_{n, m}^{\infty}\left[A_{n m}^{\mathrm{TM}} Y_{n m}^{\mathrm{TM}(1)}+A_{n m}^{\mathrm{TE}} Y_{n m}^{\mathrm{TE}(1)}\right] \tan \left(\beta_{n m}^{(1)} \frac{d_{1}}{2}\right)
\end{aligned}
$$

and

$$
Y_{\mathrm{s}}=-\mathrm{j} \sum_{n, m}^{\infty}\left[A_{n m}^{\mathrm{TM}} Y_{n m}^{\mathrm{TM}(1)}+A_{n m}^{\mathrm{TE}} Y_{n m}^{\mathrm{TE}(1)}\right] \csc \left(\beta_{n m}^{(1)} d_{1}\right) .
$$

The formulas provided for all the parameters appearing in the above expressions are frequency-dependent, which implies that the above infinite series summations have to be carried out, in principle, for every frequency value. The fact of having admittances with an involved frequency-dependent behavior does not certainly contribute to highlight the possible advantages of the equivalent circuit approach. Fortunately, for high-order harmonics $\left(\forall n, m \backslash k_{x n}^{2}+k_{y m}^{2} \gg \varepsilon_{r} k_{0}^{2}\right)$, it is possible to write

$$
\beta_{n m}^{(i)} \approx-\mathrm{j} k_{n m}=-\mathrm{j} \sqrt{k_{x n}^{2}+k_{y m}^{2}} .
$$

This means that for all the evanescent harmonics operating well below cutoff ("localized" modes in the terminology used in [23], where a related problem is dealt with), the wave admittances can be approximated as

$$
\begin{aligned}
Y_{n m}^{\mathrm{TM}(i), \text { ho }} & \approx \frac{\mathrm{j} \omega \varepsilon_{0} \varepsilon_{r}^{(i)}}{k_{n m}}=\mathrm{j} \omega C_{n m}^{(i)} \\
Y_{n m}^{\mathrm{TE}, \mathrm{ho}} & \approx \frac{1}{\frac{\mathrm{j} \omega \mu_{0}}{k_{n m}}}=\frac{1}{\mathrm{j} \omega L_{n m}}
\end{aligned}
$$

where the label "ho" indicates "high order". The $C_{n m}^{(i)}$ coefficients can be interpreted as lumped capacitances associated with sufficiently high-order TM harmonics in medium $(i)$. Similarly, $L_{n m}$ are lumped inductances associated with high-order TE harmonics (note that they do not depend on permittivities).

In the light of the above approximation, the admittances of the $\pi$-network can be decomposed in one part with certain complicated, although known, frequency dependence and another part accounting for standard capacitance and inductance admittances. Thus, assuming that there are $N_{\mathrm{TM}}$ and $N_{\mathrm{TE}}$ "low order" (lo) harmonics that do not satisfy condition (13) ("accessible modes in [23]), it is possible to write the parallel admittances in (10) and (11) in the following general form:

$$
Y_{\mathrm{p}}(\omega)=\mathrm{j} \omega C_{\mathrm{ho}}^{\mathrm{p}}+\sum_{n, m}^{N_{\mathrm{TM}}} Y_{\mathrm{lo}, n m}^{\mathrm{TM}}(\omega)+\frac{1}{\mathrm{j} \omega L_{\mathrm{ho}}^{\mathrm{p}}}+\sum_{n, m}^{N_{\mathrm{TE}}} Y_{\mathrm{lo}, n m}^{\mathrm{TE}}(\omega)
$$

where the $A_{n m}$ coefficients have been purposely incorporated to the "lo" admittances. Thus, the parallel admittances are composed of a regular parallel $L C$ tank which is connected in parallel with a few admittances having a more complex frequency dependence. Part of the admittance $Y_{\mathrm{p}}(\omega)$ is associated with the external problem $\left(Y_{\mathrm{p}}^{(0)}\right)$ and part with the internal one $\left(Y_{\mathrm{p}}^{(1)}\right)$. The series admittance in (12) can equivalently be written as

$$
Y_{\mathrm{s}}(\omega)=\sum_{n, m}^{N_{\mathrm{TM}}} Y_{\mathrm{lo}, n m}^{\mathrm{TM}}(\omega)+\sum_{n, m}^{N_{\mathrm{TE}}} Y_{\mathrm{lo}, n m}^{\mathrm{TE}}(\omega)+\mathrm{j} \omega C_{\mathrm{ho}}^{\mathrm{s}}+\frac{1}{\mathrm{j} \omega L_{\mathrm{ho}}^{\mathrm{s}}} .
$$

Nevertheless, the presence of the $\csc \left(\beta_{n m}^{(1)} d_{1}\right)$ attenuating exponential factor in (12) would allow us to suppress the effect of the series lumped elements $\left(C_{\mathrm{ho}}^{\mathrm{s}}\right.$ and $\left.L_{\mathrm{ho}}^{\mathrm{s}}\right)$ accounting for high-order mode contributions if the electric separation between metal screens is large. In such case, it is apparent that the effect of the high order harmonics can be neglected due to its residual effect. The number of modes to be retained in the description of $Y_{s}$ will obviously depend on the value of the dielectric slab thickness and should include, at least, the first propagating harmonic. The explicit expressions for all the capacitances and inductances involved in our proposed equivalent circuit are given in the Appendix.

A key point that should be noted here is that, fortunately, in many practical cases the number of frequency-dependent elements (namely, the number of "accessible harmonics) required to obtain good qualitative results is just one or two. Often, with this low number of accesible harmonics, satisfactory quantitative results can also be obtained. This makes it possible to have a "minimal" equivalent circuit whose topology is rigorously derived and its components are given in closed form. Thus, let us assume that there is only one relevant "low-order" harmonic in the frequency region of interest; i.e., a single harmonic has to be retained with its whole distributed frequency-dependence while all the others are represented by frequency-independent $L$ and $C$. This harmonic is necessarily the incident TM-polarized plane wave- which has been taken as the $\mathrm{TM}_{00}$ harmonic. In the interior region (1), this harmonic would be the only propagating harmonic with all the higher-order harmonics being below cutoff. This situation would model appropriately those configurations where the electrical distance between successive screens is large enough, say $d_{1} \gtrsim \lambda^{(1)}$, where $\lambda^{(1)}$ is the wavelength inside medium (1). In such case, the series admittance in (17) can be approximated by just one component given by

$$
Y_{\mathrm{s}}(\omega) \approx-\mathrm{j} Y_{00}^{(1)} \csc \left(\beta_{00}^{(1)} d_{1}\right)
$$

where it has been assumed that the contribution of the remaining evanescent harmonics modeled by $C_{\mathrm{ho}}^{\mathrm{s}}$ and $L_{\mathrm{ho}}^{\mathrm{s}}$ is negligible. The internal part of the parallel admittance in (11) can be rewritten as

$$
Y_{\mathrm{p}}^{(1)}=\hat{Y}_{\mathrm{p}}^{(1)}+\mathrm{j} Y_{00}^{(1)} \tan \left(\frac{\beta_{00}^{(1)} d_{1}}{2}\right)
$$




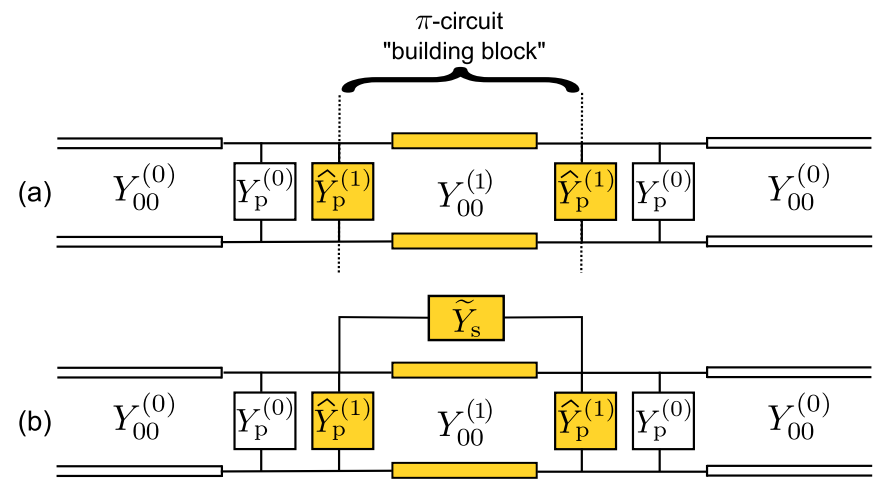

Fig. 5. Equivalent network of a pair of coupled FSS's when (a) only one loworder harmonic (the incident plane-wave) is present in the structure, and (b) there is an additional propagating low-order harmonic.

where a new parallel admittance, $\hat{Y}_{\mathrm{p}}^{(1)}$, has been introduced and defined in terms of the already known admittance $Y_{\mathrm{p}}^{(1)}$. It is easy to realize that the equivalent $\pi$-circuit of the pair of coupled FSS's in Fig. 2 can then be rephrased as the circuit shown in Fig. 5(a). The topology of this equivalent network can readily be recognized as the one usually proposed in the literature for this kind of problems when the coupling between successive screens is only accounted for by the fundamental harmonic (see, for instance, [41], [46] among many others). Although this situation is well understood, the model in Fig. 5(a) yields qualitatively and quantitatively wrong predictions in typical fishnet structures where the structured metal screens are electrically close to each other. If more than one propagation mode operates in the interior region, this simplified equivalent network is no longer valid. Nevertheless, the only significant difference in the topology required to account for interactions through higher order modes comes from the appearance of an additional series admittance, $\tilde{Y}_{\mathrm{S}}(\omega)$, as shown in Fig. 5(b). The relation between this new series admittance and $Y_{\mathrm{s}}$ in (12) is given by

$$
\tilde{Y}_{\mathrm{s}}=Y_{\mathrm{s}}+\mathrm{j} Y_{00}^{(1)} \csc \left(\beta_{00}^{(1)} d_{1}\right) \text {. }
$$

Note that the contribution to the series admittance of the propagating harmonic is explicitly extracted out in (20). Thus, $\widetilde{Y}_{\mathrm{s}}$ only contains information of the high-order modes responsible for interactions between the two metal screens. These are very few modes which are operating above cutoff (this is possible due to the presence of dielectric slabs) or below but close to their cutoff frequencies. If all the involved harmonics are far and below cutoff, using again the approximations in (13) and (14), $\widetilde{Y}_{\text {s }}$ can be interpreted as a shunt $L C$ circuit. For electrically thick dielectrics, the values of the corresponding $C$ parameter tends to be extremely small while $L$ is very large, thus giving place to a very low admittance, as expected. This means that $\widetilde{Y}_{\text {s }}$ can be neglected if the electrical thickness of the dielectric is large enough, thus explaining why the model employed in some papers dealing with stacked structures [the model in Fig. 5(a)] works properly. However, if the electric length between screens is not large enough, the more accurate model in Fig. 5(b) has to be used. In next section, it will become apparent that neglecting the role of $\widetilde{Y}_{\mathrm{s}}$ can give place to completely wrong results.

\section{Results}

In this section, the behavior of the stacked fishnet structure of interest in this work is studied under two different oper- ation regimes: 1) the "short period" regime corresponding to a longitudinal period $(d)$ less than half the transverse period $\left(P_{x}=P_{y} \equiv P\right), d<P / 2$; and 2) the "long period" regime corresponding to $d>P / 2$. Special attention is also paid to the importance of incorporating the series admittance $\widetilde{Y}_{\mathrm{s}}$ to the model-see Fig. 5(b). In the following, the electromagnetic field distribution is analyzed at the most relevant frequencies observed in the spectra to correlate them with the equivalent circuit elements presentedin the previous section. In this analysis, all the frequencies are normalized to the period; i.e., $f$ is expressed in units of $P / \lambda_{0}$ where $\lambda_{0}$ refers to the wavelength of the first Rayleigh-Wood anomaly.

\section{A. Short Longitudinal Period}

The structure analyzed in this and subsequent sections is a finite stacked fishnet lossless structure composed of five metallic screens separated by four air/dielectric layers. First, the structure is numerically analyzed by means of a full-wave Floquet-mode simulation of a unit cell using the software CST Microwave Studio. The presented numerical results come after the adaptive mesh refinement between two consecutive calculations of the scattering parameters shows differences below 0.001 .

In Fig. 6(a), the transmission coefficient under normal incidence for the short period regime $(d=0.2 P)$ with air between the perforated metal screens is shown. The dip at $f=1$ is associated with the first Rayleigh-Wood anomaly, which is the upper limit of the spectral window of interest (the diffraction regime is beyond the scope of the present work). A transmission band is clearly observed for $0.87<f<0.98$ with five recognizable peaks $\left(p_{\mathrm{s} 1}, p_{\mathrm{s} 2}, p_{\mathrm{s} 3}, p_{\mathrm{s} 4}\right.$, and $\left.p_{\mathrm{s} 5}\right)$. The relevant components $E_{y}$ and $E_{z}\left(E_{x}\right.$ is negligible $)$ of the electric field at the peak frequencies are presented in Fig. 7(a). At $p_{\mathrm{s} 1}, E_{z}$ is mainly located on the outer metal-air interfaces while $E_{y}$ is confined at the aperture, resembling the so-called ET resonance in a single layer hole array [38], [40]. Regarding the fields at the other peaks $\left(p_{\mathrm{s} 2}-p_{\mathrm{s} 5}\right)$, it can be seen that $E_{y}$ is confined near the aperture, as well. However, $E_{z}$ gradually changes: from the highest $\left(p_{\mathrm{s} 2}\right)$ to the lowest $\left(p_{\mathrm{s} 5}\right)$ frequency, $E_{z}$ becomes more and more confined between the perforated layers, where the fieldamplitude is higher. These peaks correspond with internal mode resonances [38], [54]. Furthermore, in the longitudinal direction, different distributions of maxima and minima are noticed. Interestingly, the number of variations (maxima and minima) inside the structure increases as the frequency decreases: $p_{\mathrm{s} 2}, p_{\mathrm{s} 3}, p_{\mathrm{s} 4}, p_{\mathrm{s} 5}$ corresponds to a $\lambda / 2, \lambda, 3 \lambda / 2$ and $2 \lambda$ mode inside the structure. Hence, the band has a negative-index character, as it is expected for short longitudinal periods [40], [54]. The number of these internal resonances is always equal to $N-1$, with $N$ being the number of perforated screens. Finally, on the rightmost column of Fig. 7, the $x$-component of the magnetic field, $H_{x}$, between the $3 \mathrm{rd}$ and 4 th layers is represented. The distribution of $H_{x}$ for all peaks resembles the $\mathrm{TM}_{02}$ mode of the unit-cell of the virtual parallel-plate waveguide induced by the transverse periodicity. This fact together with the $E_{z}$ distribution indicates that the relevant higher order mode involved in this transmission band is the $\mathrm{TM}_{02}$ [27], [40]. Similar field distribution is obtained between each pair of adjacent layers. This high-order dominant mode controls the values of the series admittance $\tilde{Y}_{\text {s }}$ in the circuit model in Fig. 5(b).

The equivalent circuit (EC) results for this "short-period" case are shown in Fig. 8(a). Certainly, the CPU time involved in EC calculations is completely negligible when compared with 

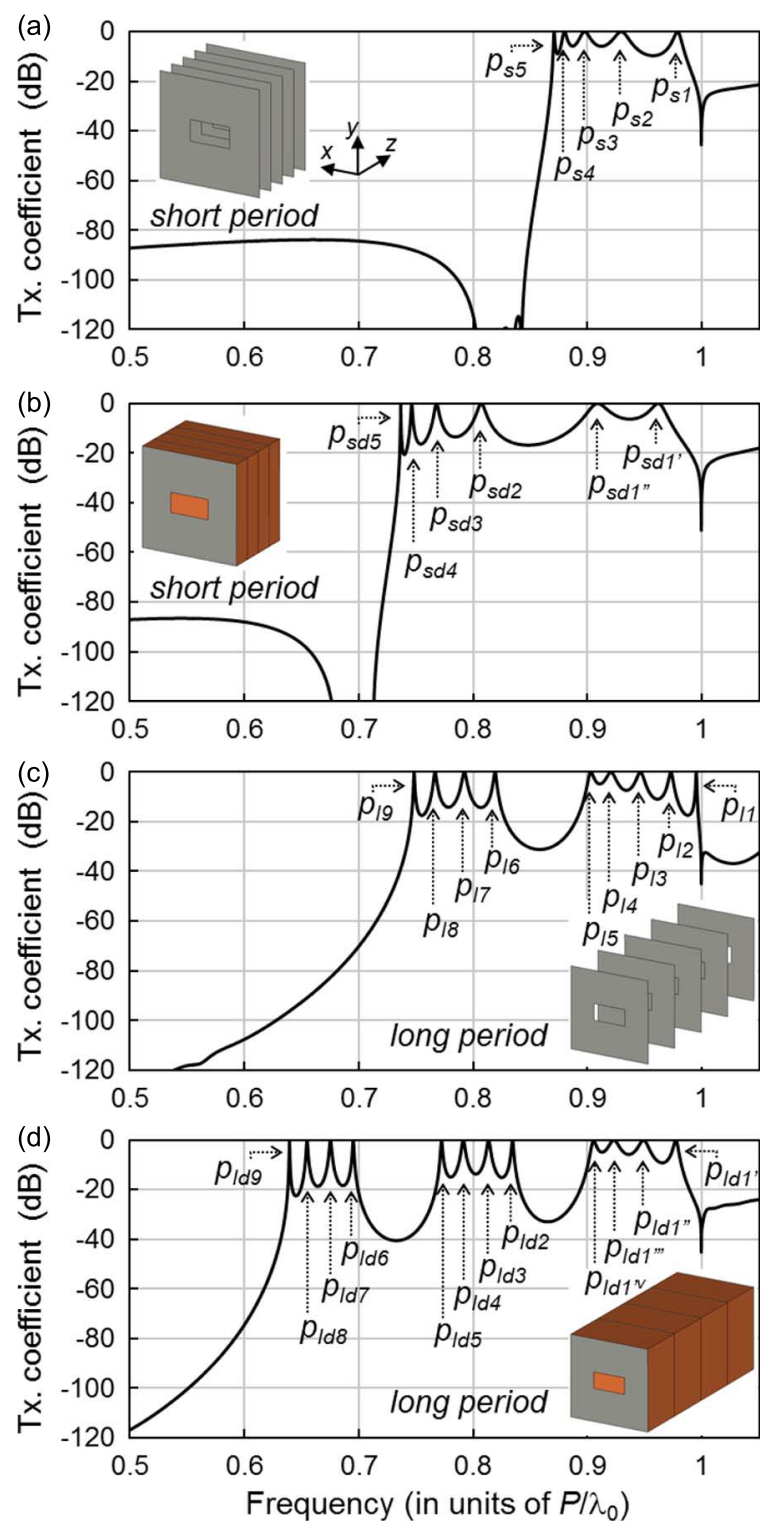

Fig. 6. Transmission coefficient of a finite fishnet structure with $N=5$ metallic screens for several configurations. $P_{x}=P_{y} \equiv P, w_{x}=0.4 P$, $w_{y}=0.2 P$; (a) $d=0.2 P$ with air between metal screens; (b) $d=0.2 P$ with dielectric $\left(\varepsilon_{r}=1.4\right)$, (c) $d=0.6 P$ with air and d) $d=0.6 P$ with dielectric between layers.

the computational effort required by the numerical approach. In this figure the full-wave simulation results [black curve] are plotted along with three curves corresponding to results of the EC approach. These three curves are labeled as $\operatorname{EC}(p, q)$, where $p$ and $q$ stand respectively for the number of low-order TE and TM harmonics (in addition to the fundamental impinging one) whose exact frequency behavior (distributed nature) is taken explicitly into account in the EC model. The rest of the high-order modes are approximated with the capacitance and inductance limits discussed in the previous section. The curve $\mathrm{EC}(0,0)$ [green line] corresponds to the topology shown in Fig. 5(b) with the coupling between the metallic screens basically accounted for by the $\mathrm{TM}_{00}$ harmonic (the $\mathrm{TE}_{00}$ harmonic is not excited in the present structure) and with $\widetilde{Y}_{\mathrm{s}}$ given by $\tilde{Y}_{\mathrm{s}}=\mathrm{j} \omega C_{\mathrm{ho}}^{\mathrm{s}}+1 /\left(\mathrm{j} \omega L_{\mathrm{ho}}^{\mathrm{s}}\right)$, after applying the simplifications in (13), (14) and (15). It should be noted that the results of this (a)
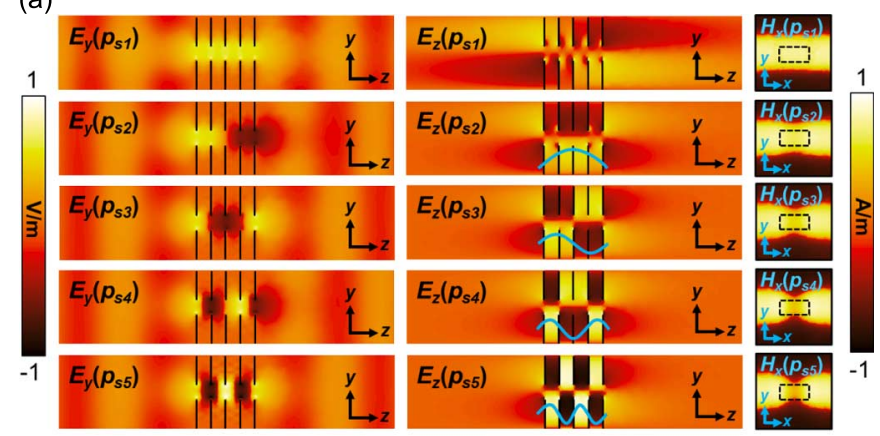

(b)
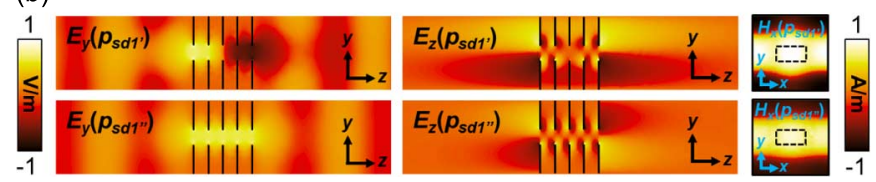

Fig. 7. Electric field $\left(E_{y}\right.$ and $\left.E_{z}\right)$ on the $y z$-plane and magnetic field $\left(H_{x}\right)$ on the $x y$-plane, at the relevant frequencies labeled in Fig. 6(a) and Fig. 6(b) for the fishnet with $d=0.2 P$ and a) with air and b) with dielectric between layers.

model and the one in which $\tilde{Y}_{\mathrm{s}}$ is taken zero are found qualitatively identical although with some quantitative discrepancies. Thus, in most practical cases, the results of the curve $\operatorname{EC}(0,0)$ can be associated with the topology of Fig. 5(a). The EC $(0,1)$ curve [cyan line] already fully corresponds to the circuit shown in Fig. 5(b), with only the first high-order TM mode $\left(\mathrm{TM}_{02}\right.$ in this case) being correctly included in the definition of $\widetilde{Y}_{\mathrm{s}}$.

It is observed that the inclusion in the circuit model of the correct frequency dependence of just the $\mathrm{TM}_{02}$ harmonic provides a good qualitative matching with the full-wave results. This is in concordance with the field distributions observed in the previous EM field inspection since high-order TM modes are responsible for such dominant $E_{z}$. Hence, in this case of screens electrically close, it is the influence of the first evanescent harmonic in the coupling between metal screens what gives place to the observed passband; i.e., the conventional connection through the fundamental harmonic as well as a wrong evaluation of the interaction due to high-order modes cannot at all account for the appearance of such band. The addition of the first high-order TE harmonic is necessary for a better quantitative agreement, as shown by the red curve EC $(1,1)$. Indeed, this harmonic has the same cutoff frequency as the $\mathrm{TM}_{02}$ but its influence on the overall field is smaller (since the reaction of this eigenfield with the assumed aperture field is smaller too). In the EM field inspection, the TE distribution is mainly masked. However, by looking at the magnetic field on the cross-sectional $x z$-plane in Fig. 9, we can observe some $H_{z}$ which accounts for the inductive coupling between holes [45], particularly at $p_{\mathrm{s} 1}$. Clearly the agreement between the circuit model and the numerical results can be systematically improved by adding higher-order modes to the definition of $\widetilde{Y}_{\mathrm{s}}$.

Fishnet metamaterials usually employ dielectric slabs between the metallic layers for the sake of robustness and ease of fabrication. In Fig. 6(b) the numerically computed transmission coefficient for the short period case with a dielectric is presented. The transmission band now extends from $f=0.74$ to $f=0.97$, which indicates that adding the dielectric widens the operation bandwidth. In addition, six peaks $\left(p_{\mathrm{sd} 1^{\prime}}, p_{\mathrm{sd} 1^{\prime \prime}}\right.$, $p_{\mathrm{sd} 2}, p_{\mathrm{sd} 3}, p_{\mathrm{sd} 4}$ and $\left.p_{\mathrm{sd} 5}\right)$ are present. Looking at the field distribution for $p_{\mathrm{sd} 1^{\prime}}$ and $p_{\mathrm{sd} 1^{\prime \prime}}$, shown in Fig. $7(\mathrm{~b})$, a clear 

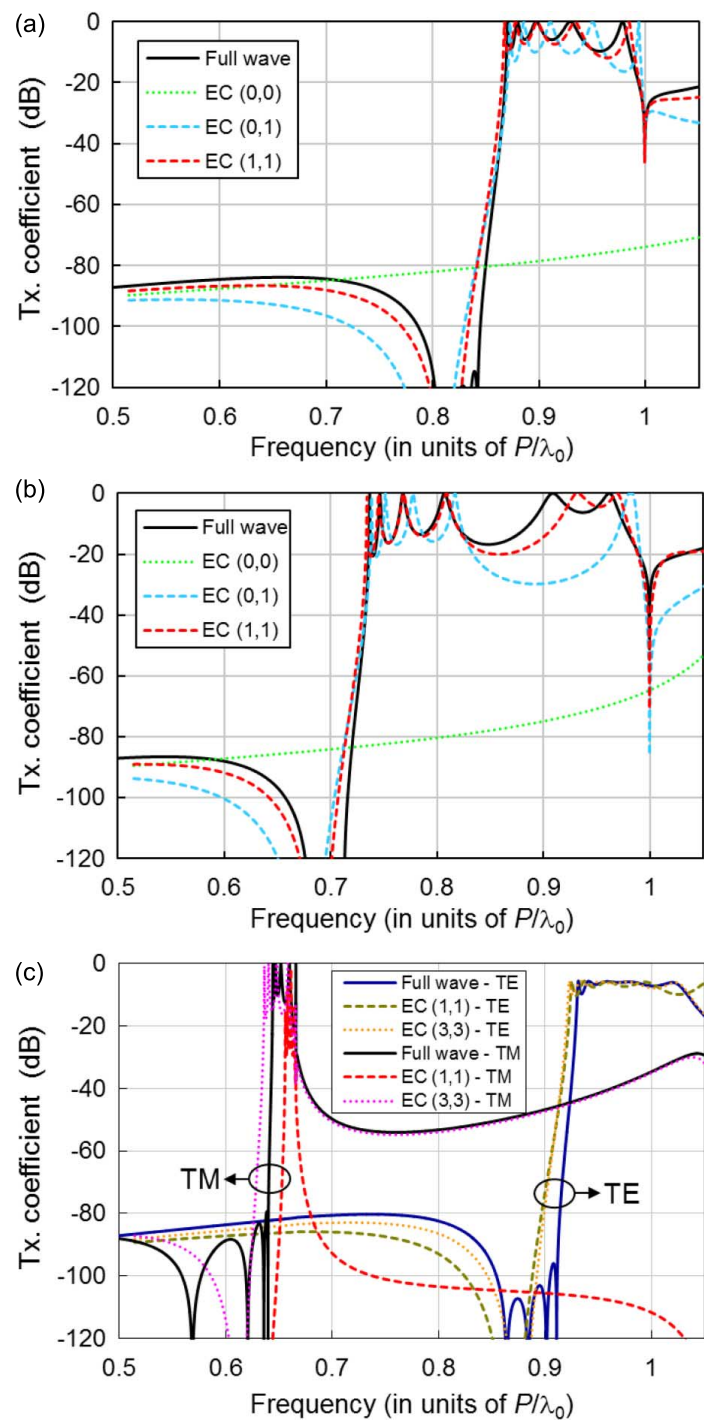

Fig. 8. EC results for the "short-period" structures previously analyzed in Figs. 6(a) and (b). (a) Air between metal screens; (b) dielectric material between metal layers. (c) Oblique incidence $\left(\theta=30^{\circ}\right)$, with the electric field directed along the $y$ direction, for the structure in (a). This figure (c) also shows EC results when a relatively high number of harmonics are explicitly considered.

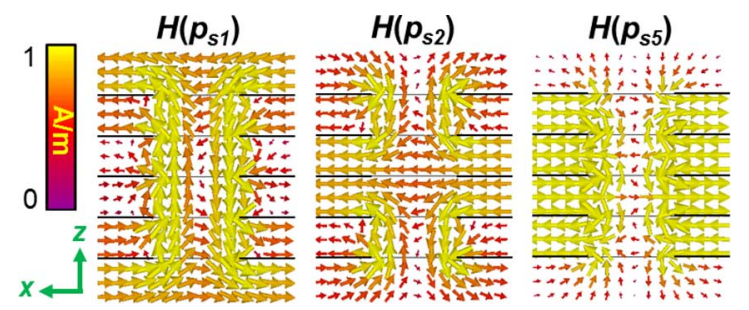

Fig. 9. Magnetic field on the $x z$-plane in the middle plane of the unit cell at the relevant frequencies $p_{\mathrm{s} 1}, p_{\mathrm{s} 2}$ and $p_{\mathrm{s} 5}$.

concordance with the fields at $p_{\mathrm{s} 1}$ (case without dielectric) is observed. Actually $p_{\mathrm{s} 1}$ and $p_{\mathrm{sd} 1}{ }^{\prime \prime}$ present the same distribution in both $E_{y}$ and $E_{z}$ and therefore $p_{\mathrm{sd} 1}$ " is identified as the even external ET resonance. For $p_{\mathrm{sd} 1^{\prime}}$, the $E_{z}$ is mainly located on the outer metal-air interfaces and $E_{y}$ is highly confined at the aperture; although a $\lambda / 2$ longitudinal resonance is observed in this case. The symmetric distribution of $E_{z}$ with respect to the transversal central $x y$-plane at the external faces indicates that $p_{\mathrm{sd} 1^{\prime}}$ can be associated with the so-called odd external ET resonance [38]. The magnetic field distribution also shows the excitation of the $\mathrm{TM}_{02}$ higher order mode. The field distributions for $p_{\mathrm{sd} 2}-p_{\mathrm{sd} 5}$ are omitted in Fig. 7(b) since they are similar to the cases already studied in $p_{\mathrm{s} 2}-p_{\mathrm{s} 5}$.

This case can also be conveniently handled with the EC approach, as it is shown in Fig. 8(b). If the series interaction associated with the presence of a non-vanishing $\widetilde{Y}_{\mathrm{S}}$ is eliminated or incorrectly accounted for [case $(0,0)$, green line], the circuit model has not any physical meaning again. It is then essential to correctly introduce the interaction between successive perforated screens through, at least, the first high-order mode $\left(\mathrm{TM}_{02}\right.$ in this structure) to account for the existence of transmission peaks in the frequency region of interest [cyan curve in Fig. 8(b)]. The proper inclusion of the contribution of the $\mathrm{TM}_{02}$ in $\widetilde{Y}_{\mathrm{s}}$ explains the appearance of the transmission band and yields reasonably accurate results for the low-frequency part of the transmission band. However, it is clear that quantitative agreement is poor, especially as the frequency increases, and even one of the transmission peaks is lost. This drawback is alleviated by adding to $\widetilde{Y}_{\mathrm{S}}$ the contribution of the first TE mode too [red line in Fig. 8(b)]. Similar to the non-dielectric case, TE modes are less excited but still noticeable in the field inspection, particularly at $p_{\mathrm{sd} 1^{\prime}}$ and $p_{\mathrm{sd} 1^{\prime \prime}}$ which, in the EC results, appear when the $\mathrm{TE}_{20}$ dispersion is taken account. More modes should be added for a better quantitative agreement but the model would become more and more cumbersome. Actually, the curve corresponding to $\mathrm{EC}(1,2)$ has been found to be almost indistinguishable in the graphic to that corresponding to CST Microwave Studio. With a computation involving EC $(3,3)$, the numerical agreement with CST Microwave Studio is very good. It is worth mentioning that the circuit-model approach provides a simple explanation for the difference between the transmission patterns observed for the structure with and without dielectrics. When the space between metal screens is occupied by air, the first two high-order modes involved in the interaction are below cutoff in the whole frequency range of interest. However, when the dielectrics are present, the cutoff for the two lowest order TM and TE modes is approximately at $f=0.845$, in such a way that the frequency dependence of $\widetilde{Y}_{\mathrm{S}}$ drastically changes due to the propagation of the first two high-order modes, thus allowing for additional transmission peaks. Finally, Fig. 8(c) shows the results obtained for oblique TE/TM incidence and for an azhimutal angle $\theta=30^{\circ}$. In this figure, the equivalent-circuit curve corresponding to $\mathrm{EC}(1,1)$ means that all the $(m, n) \mathrm{TM} / \mathrm{TE}$ harmonics involving $m, n=-1,0,1$ are explicitly considered in the dynamic series [for normal incidence, there appears degeneracy in the harmonics of the type $( \pm m, \pm n)]$. These EC $(1,1)$ data show a good qualitative behavior but the figure also makes it apparent that for a good quantitative behavior it is necessary to explicitely consider more harmonics. Actually, a satisfactory quantitative behavior is again achieved with $\mathrm{EC}(1,2)$ as in Fig. 8(b) [relative errors around or less than 1\%], and numerical excellent agreement is obtained for the TE polarization for $\mathrm{EC}(3,3)$. Anyway, this figure clearly demonstrate the ability of the EC approach to deal also with oblique incidence reliably, without a significant increase of the required CPU time (the two thousand frequency points shown in Fig. 8(c) for $\mathrm{EC}(3,3)$ were computed in about 1 to 2 seconds on an i7 laptop).

\section{B. Long Longitudinal Period}

In the long period case, (a longitudinal periodicity of $d=$ $0.6 P$ is assumed), considering air between the metallic layers, 
a significant change in the transmission coefficient is appreciated in Fig. 6(c). Now, two different transmission bands are observed. The higher band is located between $f=0.9$ and $f$ $=0.99$ and five peaks are noticed $\left(p_{11}, p_{12}, p_{13}, p_{14}\right.$, and $\left.p_{15}\right)$. By examining the fields at these peaks in Fig. 10(a), the nature of this band can be immediately extracted. At $p_{11}, E_{y}$ and $E_{z}$ corresponds with the fields already observed at $p_{\mathrm{s} 1}$ (i.e., the even external ET resonance). Likewise, the fields at $p_{15}$ also resemble the fields observed at $p_{\mathrm{s} 5}$ and, therefore, it is related to internal mode resonances as well. Due to the similarity between the field for the short and long period cases, the fields at $p_{12}$ to $p_{14}$ are omitted. The lower band extends from $f=0.75$ to $f$ $=0.82$ and it contains four peaks $\left(p_{16}, p_{17}, p_{18}\right.$, and $\left.p_{19}\right)$. Analyzing the fields at the peaks, a completely different distribution is noticed [Fig. 10(a)]. The $E_{z}$ component is no longer the dominant contribution since the mutual coupling between adjacent layers is partially lost due to the larger longitudinal periodicity. Now, the highest intensity of the electric field is observed in $E_{y}$. This kind of field distribution is well known in stacked structures and corresponds with Fabry-Pérot resonances [40], [46], [54]. Moreover, peaks at higher frequencies show more maxima and minima (contrarily to what happens in the higher band) and, therefore, this is a band with positive index of refraction [40], [54]. By examining the magnetic field between the layers $\left[H_{x}\left(p_{16}\right)\right.$ to $\left.H_{x}\left(p_{19}\right)\right], H_{x}$ does not show a relevant variation along the $y$-axis, differently from the previous bands. The continuous distribution of $H_{x}$ and $E_{y}$ along with the dominant character of the latter over $E_{z}$ resemble the TEM mode, and excitation of higher order modes are not observed. As discussed in [54], the Fabry-Pérot band for short periods $(d<\lambda / 2)$ takes place above the Rayleigh-Wood's anomaly. Hence, it was not observed in the previous analysis.

It is interesting now to find out what the proposed EC model predicts for this situation. The results plotted in Fig. 11(a) show that the basic circuit $[\mathrm{EC}(0,0)$ : green curve], where interaction between metal layers is carried out through the fundamental TEM mode, qualitatively accounts for the first transmission band. This model also predicts the appearance of a second transmission band at about twice the frequency range of the first transmission band, but this happens beyond the onset of the diffraction regime. For structures with an electrically-small transverse period, such as those studied in [41], the simplified $\mathrm{EC}(0,0)$ model has proven to give very good results. However, in the present situation, the transverse period is not so small and the influence of the interaction through high-order modes gives place to additional bands of transparency below the Rayleigh-Wood frequency point $(f=1)$. Thus, the existence of the second transmission band observed in Fig. 11(a) is found to be intimately linked with the existence of a path of interaction due to high-order modes, which are purely evanescent in this frequency range for air filling. As the separation between screens is now relatively large, the accuracy of the model is excellent when only the full contribution of the first high-order TM and TE modes are included in $\widetilde{Y}_{\mathrm{s}}$. This agreement is now better than in the short period case because, for the present separation between layers, the interaction through modes with even higher order is completely negligible.

Finally, the fishnet configuration corresponding to the long period and dielectric slabs with permittivity $\varepsilon_{r}=1.4$ between the metallic layers is analyzed. Here it is observed the appearance of three transmission bands in Fig. 6(d) instead of two. The highest frequency band extends from $f=0.9$ to $f=0.98$ (peaks $p_{\mathrm{ld} 1^{\prime}}, p_{\mathrm{ld} 1^{\prime \prime}}, p_{\operatorname{ld} 1^{\prime \prime \prime}}$ and $p_{\operatorname{ld} 1^{\prime v}}$ ), the central one from $f$
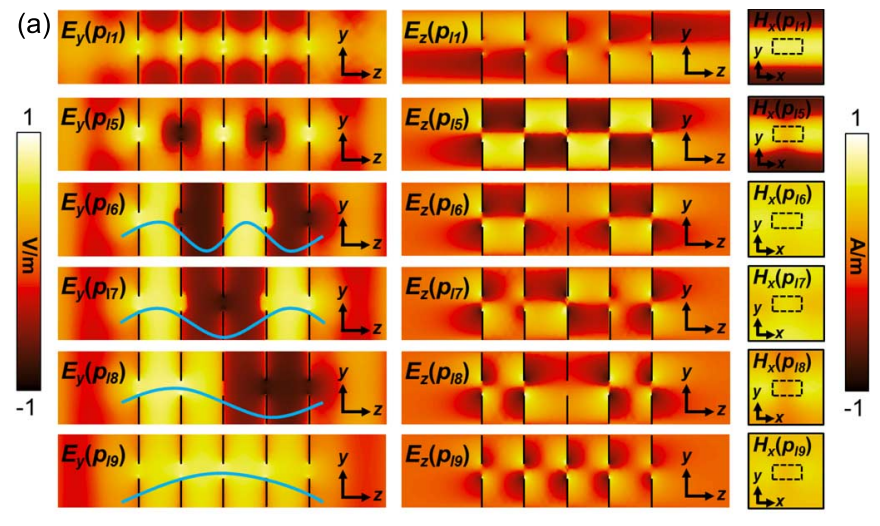

(b)

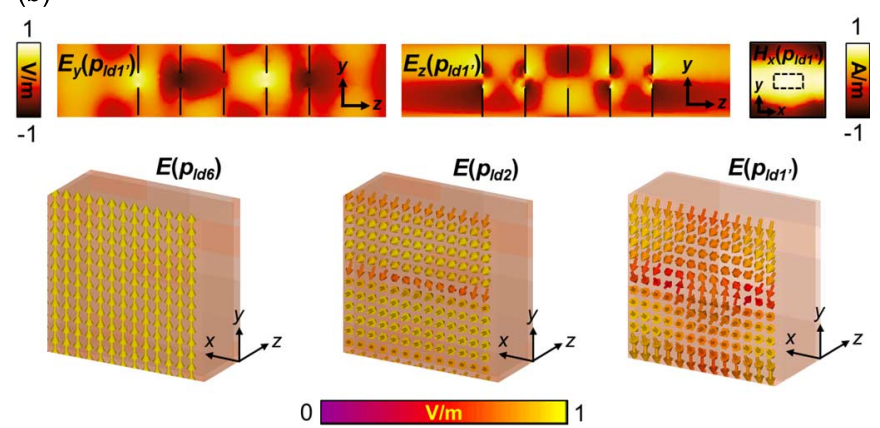

Fig. 10. Electric field $\left(E_{y}\right.$ and $\left.E_{z}\right)$ on the $y z$-plane and magnetic field $\left(H_{x}\right)$ on the $x y$-plane, at the relevant frequencies labeled in Fig. 6(c) and Fig. 6(d) for the fishnet with $d=0.6 P$ and a) with air and b) with dielectric between layers. Bottom figures in $b$ ) also show the perspective view of the electric field on the xy-plane.

$=0.77$ to $f=0.83$ (peaks $p_{\mathrm{ld} 2}, p_{\mathrm{ld} 3}, p_{\mathrm{ld} 4}$ and $\left.p_{\mathrm{ld} 5}\right)$ and the lower one from $f=0.64$ to $f=0.69$ (peaks $p_{\operatorname{ld} 6}, p_{\mathrm{ld} 7}, p_{\mathrm{ld} 8}$ and $\left.p_{\mathrm{ld} 9}\right)$. In the lower panels of Fig. 10(b), the electric field in a transversal $x y$-plane between the $3 \mathrm{rd}$ and 4 th layer is depicted for one peak of every band: $E\left(p_{\operatorname{ld} 6}\right), E\left(p_{\operatorname{ld} 2}\right)$, and $E\left(p_{\operatorname{ld} 1^{\prime}}\right)$. The dominant components of the fields within all peaks in a band are very similar, so these peaks are taken as representative of the fields in each band. The fields at the lower $\left[E\left(p_{\operatorname{ld} 6}\right)\right]$ and the central $\left[E\left(p_{\mathrm{ld} 2}\right)\right]$ bands correspond with the fields obtained in the lower and higher bands, respectively, in the free-standing long-period fishnet. At $p_{\mathrm{ld} 6}$ the electric field corresponds to a Fabry-Pérot resonance since it points primarily to the $y$-axis. On the other hand, at $p_{\mathrm{ld} 2}$ the electric field is mainly longitudinal pointing to opposite directions in the upper and lower part of the unit cell. Hence, the peaks are identified as the internal mode resonances associated with the $\mathrm{TM}_{02}$ harmonic. Similarly, the fact that $E\left(p_{\mathrm{ld} 1^{\prime}}\right)$ also points like $E\left(p_{\mathrm{ld} 2}\right), E_{y}$ is highly confined at the apertures, and the presence of $E_{z}$ primarily external to the whole structure suggest that this band also corresponds with external resonances ascribed to the high-order $\mathrm{TM}_{02}$ mode. Besides, this band exhibits positive index of refraction. The appearance and bandwidth of this new band is highly dependent of the longitudinal periodicity and the permittivity of the dielectric and hence it is not always present.

Despite this complex behavior, our circuit modeling shown in Fig. 11(b) is able to predict the behavior of this band without including additional circuit elements with respect to the non-dielectric fishnet. The relatively trivial model denoted as $\mathrm{EC}(0,0)$ predicts the existence of the first transmission band but completely loses the information of the interactions that gives place to the two additional transmission bands appearing in the non- 

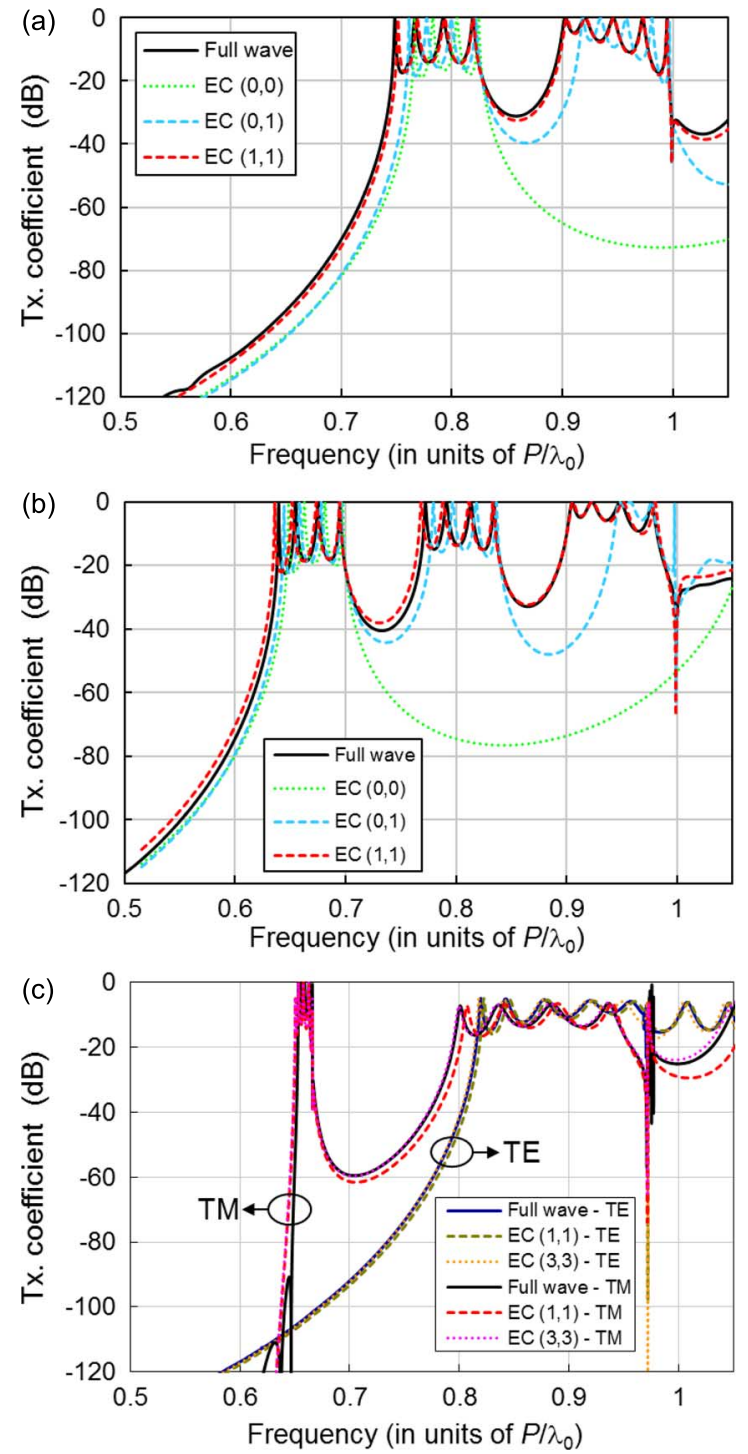

Fig. 11. (a) and (b): Equivalent circuit (EC) results for the "long-period" structures previously analyzed in Fig. 6(c) and (d), respectively. (c) Oblique incidence $\left(\theta=30^{\circ}\right)$, with the impinging electric field directed along the $y$ direction, for the structure in (a). This figure (c) also shows EC results when a relatively high number of harmonics are explicitly considered.

diffraction region. The inclusion in $\widetilde{Y}_{\mathrm{s}}$ of the interaction through the dominant high-order TM mode is already enough to account for the existence of those two high-frequency bands, one below the cutoff of all the high-order modes (thus implying interaction by means of evanescent fields) and the other above cutoff. However, for the correct prediction of the number of peaks in the third transmission band, the influence of the TE mode must be taken into account [red curve].

In Fig. 11(c) it is finally depicted the case of oblique incidence, which similarly to Fig. 8(c) also shows good agreement between our EC results and CST Microwave Studio. Actually, for this longer period case, it is found a satisfactory quantitative agreement for the case $\mathrm{EC}(1,1)$ and excellent matching for the case $\operatorname{EC}(3,3)$.

\section{CONCLUSION}

This paper discusses the behavior of NIM implemented as stacked identical metal screens with 2D periodic distributions of subwavelength apertures (the so-called fishnet structures). A first analysis of the structure is carried out by means of a numerically intensive electromagnetic solver. It is demonstrated next that the equivalent circuit approach, commonly used in the analysis of frequency selective surfaces, yields a faster solving procedure which also sheds light on the physics behind the appearance of the different observed transmission bands. In contrast with many analytical models available in the specialized literature, the proposed model accounts for the frequency-dependent behavior of the contributions associated with the lowest-order excited modes and, most importantly, correctly accounts for the high-order mode interaction between successive screens. The latter fact is very important since, for typical dimensions involved in the implementation of stacked fishnet structures, high-order mode interaction is key to explain the existence of some of the transmission bands. The model automatically collapses into simpler conventional circuits involving interaction through the fundamental TEM wave if the distance between screens and/or the transverse period have the appropriate values. The accuracy of the circuit model can be systematically enhanced by adding high-order contributions at the expenses of generating a more complex circuit topology. In any case, the numerical effort demanded by these circuit approaches is negligible when compared with usual numerical codes.

\section{APPENDIX}

In the computation of the numerical results in this work, the following approximated expression is used for the spatial profile of the aperture field [53]:

$$
E_{\mathrm{a}}(x, y) \propto \cos \left(\frac{\pi x}{w_{x}}\right)\left[1-\left(\frac{2 x}{w_{x}}\right)^{2}\right]^{-1 / 2}
$$

whose Fourier transform is given by

$$
\begin{aligned}
\widetilde{E}_{\mathrm{a}}\left(k_{x n}, k_{y m}\right) \propto[ & {\left[J_{0}\left(\left|k_{x n} w_{x} / 2+\pi / 2\right|\right)\right.} \\
& \left.+J_{0}\left(\left|k_{x n} w_{x} / 2-\pi / 2\right|\right)\right] \frac{\sin \left(\frac{k_{y m} w_{y}}{2}\right)}{k_{y m}}
\end{aligned}
$$

where $J_{0}$ stands for the zeroth-order Bessel function of the first kind. Using this aperture field profile, analytical expressions for the high-order capacitances and inductances in the model can be written explicitly in terms of the structure parameters. Thus, given $N$ and $M$ such that the high-order condition $\beta_{n m}^{(i)} \approx$ $-\mathrm{j} k_{n m}$ holds for $n>N$ or $m>M$, the following expressions are found for the external parallel elements of the $\pi$-network [see (10) and (16)]:

$$
\begin{aligned}
& C_{\mathrm{ho}}^{\mathrm{p}(0)}=\varepsilon_{0} w_{y} \frac{\left(\frac{P_{y}}{w_{y}}\right)^{3}}{2 \pi^{3} J_{0}^{2}\left(\frac{\pi}{2}\right)} \sum_{\text {ho }}^{\infty} \frac{F_{n m}^{2}}{\left[\left(\frac{P_{y}}{P_{x}} n\right)^{2}+m^{2}\right]^{\frac{3}{2}}} \\
& L_{\mathrm{ho}}^{\mathrm{p}(0)}=\mu_{0} w_{y} \frac{\pi J_{0}^{2}\left(\frac{\pi}{2}\right) w_{y} P_{x}}{2 P_{y}^{2}} \frac{1}{\sum_{\text {ho }}^{\infty} \frac{n^{2} F_{n m}^{2}}{m^{2}\left[\left(\frac{P_{y}}{P_{x}} n\right)^{2}+m^{2}\right]^{\frac{1}{2}}}}
\end{aligned}
$$

$$
\begin{aligned}
& F_{n m}^{\text {with }}=\left[J_{0}\left(\left|n \pi \frac{w_{x}}{P_{x}}+\frac{\pi}{2}\right|\right)+J_{0}\left(\left|n \pi \frac{w_{x}}{P_{x}}-\frac{\pi}{2}\right|\right)\right] \\
& \times \sin \left(m \pi \frac{w_{y}}{P_{y}}\right)
\end{aligned}
$$


and where the sums extend to all $n, m$ such that $n>N$ or $m>$ $M$. Concerning the internal parallel element of the $\pi$-network [(11) and (16)], the $C_{\mathrm{ho}}^{\mathrm{p}(1)}$ and $L_{\mathrm{ho}}^{\mathrm{p}(1)}$ values can be obtained from (23) and (24) just by replacing $F_{n m}^{2}$ with

$$
F_{n m}^{\prime 2}=F_{n m}^{2} \tanh \left(\pi\left[\left(\frac{d_{1}}{P_{x}} n\right)^{2}+\left(\frac{d_{1}}{P_{y}} m\right)^{2}\right]^{\frac{1}{2}}\right)
$$

and multiplying (23) by $\varepsilon_{r}^{(1)}$. Note that the tanh function quickly approaches unity for values of its argument larger than a few units. Therefore, for largely spaced screens the value of the sums can be taken directly equal to those in (23) and (24), which is a consequence of the fact that largely spaced screens do not interact through evanescent harmonics. For closely spaced screens, only a few addends in the infinite sum (those with lower values of $n$ and $m$ ) are significantly different from those in (23) and (24). Similarly, the expressions of $C_{\text {ho }}^{\text {s }}$ and $L_{\mathrm{ho}}^{\mathrm{s}}$ in the series element [(12) and (17)] can be obtained from (23) and (24) by replacing $F_{n m}^{2}$ with

$$
F_{n m}^{\prime \prime 2}=F_{n m}^{2} \operatorname{csch}\left(2 \pi\left[\left(\frac{d_{1}}{P_{x}} n\right)^{2}+\left(\frac{d_{1}}{P_{y}} m\right)^{2}\right]^{\frac{1}{2}}\right)
$$

and multiplying (23) by $\varepsilon_{r}^{(1)}$. In this case, due to the fast exponential decrease of the csch function, the contribution of the high-order terms to the series element can be neglected for largely spaced screens, whereas for closely spaced screens only the first few addends contribute significantly to the final values of the high-order capacitance and inductance. Finally, it is worth noting that the following asymptotic form:

$$
J_{0}\left(x+\frac{\pi}{2}\right)+J_{0}\left(x-\frac{\pi}{2}\right) \approx \sqrt{\frac{\pi}{2}} \frac{\cos (x-3 \pi / 4)}{x^{\frac{3}{2}}}
$$

can be conveniently used for $x \gtrsim 10$.

\section{REFERENCES}

[1] R. Marqués, F. Martín, and M. Sorolla, Metamaterials With Negative Parameters: Theory, Design, Microwave Applications. Hoboken, NJ: Wiley, 2008.

[2] L. Solymar and E. Shamonina, Waves in Metamaterials. New York: Oxford Univ. Press, 2009.

[3] R. A. Shelby, D. R. Smith, and S. Schultz, "Experimental verification of a negative index of refraction," Science, vol. 292, no. 5514, pp. 77-79, Apr. 2001.

[4] R. S. Penciu, M. Kafesaki, T. Koschny, E. N. Economou, and C. M. Soukoulis, "Magnetic response of nanoscale left-handed metamaterials," Phys. Rev. B, vol. 81, no. 23, p. 235111, Jun. 2010.

[5] C. García-Meca, J. Hurtado, J. Martí, A. Martínez, W. Dickson, and A. V. Zayats, "Low-loss multilayered metamaterial exhibiting a negative index of refraction at visible wavelengths," Phys. Rev. Lett., vol. 106, no. 6, p. 067402 , Feb. 2011.

[6] T. W. Ebbesen, H. J. Lezec, H. F. Ghaemi, T. Thio, and P. A. Wolff, "Extraordinary optical transmission through sub-wavelength hole arrays," Nature, vol. 391, no. 6668, pp. 667-669, Feb. 1998.

[7] M. Beruete, M. Sorolla, I. Campillo, J. S. Dolado, L. Martín-Moreno, J. Bravo-Abad, and F. J. García-Vidal, "Enhanced millimeter-wave transmission through subwavelength hole arrays," Opt. Lett., vol. 29, no. 21, pp. 2500-2502, Nov. 2004.

[8] M. Beruete, M. Sorolla, and I. Campillo, "Left-handed extraordinary optical transmission through a photonic crystal of subwavelength hole arrays," Opt. Express, vol. 14, no. 12, pp. 5445-5455, Jun. 2006.

[9] M. Navarro-Cía, M. Beruete, M. Sorolla, and I. Campillo, "Negative refraction in a prism made of stacked subwavelength hole arrays," Opt. Express, vol. 16, no. 2, pp. 560-566, Jan. 2008.
[10] M. Navarro-Cía, M. Beruete, F. Falcone, J. M. Illescas, I. Campillo, and M. Sorolla, "Mastering the propagation through stacked perforated plates: Subwavelength holes vs. propagating holes," IEEE Trans. Antennas Propagat., vol. 59, no. 8, pp. 2980-2988, Aug. 2011.

[11] S. Zhang, W. Fan, N. Panoiu, K. Malloy, R. Osgood, and S. Brueck, "Experimental demonstration of near-infrared negative-index metamaterials," Phys. Rev. Lett., vol. 95, no. 13, pp. 1-4, Sep. 2005.

[12] G. Dolling, C. Enkrich, M. Wegener, C. M. Soukoulis, and S. Linden, "Simultaneous negative phase and group velocity of light in a metamaterial," Science, vol. 312, no. 5775, pp. 892-894, May 2006.

[13] M. Beruete, M. Sorolla, M. Navarro-Cía, and I. Campillo, "Polarized left-handed extraordinary optical transmission of subterahertz waves," Opt. Express, vol. 15, no. 13, pp. 8125-8134, Jun. 2007.

[14] M. Beruete, M. Navarro-Cía, M. Sorolla, and I. Campillo, "Planoconcave lens by negative refraction of stacked subwavelength hole arrays," Opt. Express, vol. 16, no. 13, pp. 9677-9683, Jun. 2008.

[15] M. Navarro-Cía, M. Beruete, I. Campillo, and M. Sorolla, "Beamforming by left-handed extraordinary transmission metamaterial biand plano-concave lens at millimeter-waves," IEEE Trans. Antennas Propagat., vol. 59, no. 6, pp. 2141-2151, Jun. 2011.

[16] M. Navarro-Cía, M. Beruete, I. Campillo, and M. Sorolla, "Millimeter-wave left-handed extraordinary transmission metamaterial demultiplexer," IEEE Antennas Wirel. Propagat. Lett., vol. 8, pp. 212-215, 2009.

[17] M. Beruete, M. Navarro-Cía, F. Falcone, I. Campillo, and M. Sorolla, "Single negative birefringence in stacked spoof plasmon metasurfaces by prism experiment," Opt. Lett., vol. 35, no. 5, pp. 643-645, Mar. 2010.

[18] R. Ulrich, "Far-infrared properties of metallic mesh and its complementary structure," Infrared Phys., vol. 7, pp. 37-55, 1967.

[19] I. Palocz and A. A. Oliner, "Equivalent network of a multimode planar grating," IEEE Trans. Microw. Theory Techn., vol. 18, no. 5, pp. 244-252, May 1970.

[20] C. K. Lee and R. J. Langley, "Equivalent-circuit models for frequencyselective surfaces at oblique angles of incidence," IEE Proc. Microw., Antennas Propagat., vol. 132, no. 6, pp. 395-399, Oct. 1985.

[21] B. Munk, Frequency Selective Surfaces: Theory and Design. New York: Wiley, 2000.

[22] R. Dubrovka, J. Vazquez, C. Parini, and D. Moore, "Equivalent circuit method for analysis and synthesis of frequency selective surfaces," IEE Proc. Microw., Antennas Propagat., vol. 153, no. 3, pp. 213-220, March 2006.

[23] S. Monni, G. Gerini, A. Neto, and A. G. Tijhuis, "Multi-mode equivalent networks for the design and analysis of frequency selective surfaces," IEEE Trans. Antennas Propagat., vol. 55, no. 10, pp. 2824-2835, Oct. 2007.

[24] M. García-Vigueras, F. Mesa, F. Medina, R. Rodríguez-Berral, and J. L. Gómez-Tornero, "Simplified circuit model for metallic arrays of patches sandwiched between dielectric slabs under arbitrary incidence," IEEE Trans. Antennas Propagat., vol. 60, no. 10, pp. 4637-4649, Oct. 2012.

[25] D. Cavallo, W. H. Syed, and A. Neto, "Closed-form analysis of artificial dielectric layers-Part I: Properties of a single layer under planewave incidence," IEEE Trans. Antennas Propag., vol. 62, no. 12, pp. 6256-6264, Dec. 2014.

[26] D. Cavallo, W. H. Syed, and A. Neto, "Closed-form analysis of artificial dielectric layers-Part I: Part II: Extension to multiple layers and arbitrary ilumination," IEEE Trans. Antennas Propag., vol. 62, no. 12, pp. 6265-6273, Dec. 2014.

[27] F. Medina, F. Mesa, and R. Marqués, "Extraordinary transmission through arrays of electrically small holes from a circuit theory perspective," IEEE Trans. Microw. Theory Techn., vol. 56, no. 12, pp. 3108-3120, Dec. 2008.

[28] N. Marcuvitz, Waveguide Handbook, ser. MIT Radiat. Lab. Series. New York: McGraw-Hill/IEE/Peregrinus, 1986, vol. 10.

[29] F. Medina, F. Mesa, and D. C. Skigin, "Extraordinary transmission through arrays of slits: A circuit theory model," IEEE Trans. Microw. Theory Techn., vol. 58, no. 1, pp. 105-115, Jan. 2010.

[30] V. Torres, R. Ortuño, P. Rodríguez-Ulibarri, A. Griol, A. Martínez, M. Navarro-Cía, M. Beruete, and M. Sorolla, "Mid-infrared plasmonic inductors: Enhancing inductance with meandering lines," Sci. Rep., vol. 4, no. 3592, pp. 1-4, Jan. 2014.

[31] E. Yarmoghaddam, G. K. Shirmanesh, A. Khavasi, and K. Mehrany, "Circuit model for periodic array of slits with multiple propagating diffracted orders," IEEE Trans. Antennas Propagat., vol. 62, no. 8, pp. 4041-4048, Aug. 2014. 
[32] M. Beruete, M. Navarro-Cía, S. A. Kuznetsov, and M. Sorolla, "Circuit approach to the minimal configuration of terahertz anomalous extraordinary transmission," Appl. Phys. Lett., vol. 98, no. 1, p. 014106, Jan. 2011.

[33] M. Beruete, M. Navarro-Cía, and M. Sorolla, "Understanding anomalous extraordinary transmission from equivalent circuit and grounded slab concepts," IEEE Trans. Microw. Theory Techn., vol. 59, no. 9, pp. 2180-2188, Sep. 2011.

[34] R. Yang, R. Rodríguez-Berral, F. Medina, and Y. Hao, “Analytical model for the transmission of electromagnetic waves through arrays of slits in perfect conductors and lossy metal screens," J. Appl. Phys., vol. 109, no. 10, p. 103107, May 2011.

[35] R. Rodriguez-Berral, C. Molero, F. Medina, and F. Mesa, "Analytical wideband model for strip/slit gratings loaded with dielectric slabs," IEEE Trans. Microw. Theory Techn., vol. 60, no. 12, pp. 3908-3918, Dec. 2012.

[36] M. Navarro-Cía, P. Rodríguez-Ulibarri, V. Torres, and M. Beruete, "Quarter-wave plate based on dielectric-enabled extraordinary resonant transmission," IEEE Photon. Technol. Lett., vol. 24, no. 11, pp. 945-947, Jun. 2012.

[37] A. Mary, S. Rodrigo, F. Garcia-Vidal, and L. Martin-Moreno, "Theory of negative-refractive-index response of double-fishnet structures," Phys. Rev. Lett., vol. 101, no. 10, pp. 2-5, Sep. 2008.

[38] R. Ortuño, C. García-Meca, F. Rodríguez-Fortuño, J. Martí, and A. Martínez, "Role of surface plasmon polaritons on optical transmission through double layer metallic hole arrays," Phys. Rev. B, vol. 79, no. 7, pp. 1-10, Feb. 2009.

[39] J. Yang, C. Sauvan, H. T. Liu, and P. Lalanne, "Theory of fishnet negative-index optical metamaterials," Phys. Rev. Lett., vol. 107, no. 4, p. 043903, Jul. 2011.

[40] M. Beruete, I. Campillo, M. Navarro-Cía, F. Falcone, and M. Sorolla Ayza, "Molding left- or right-handed metamaterials by stacked cutoff metallic hole arrays," IEEE Trans. Antennas Propagat., vol. 55, no. 6 , pp. 1514-1521, Jun. 2007.

[41] C. S. R. Kaipa, A. B. Yakovlev, F. Medina, F. Mesa, C. A. M. Butler, and A. P. Hibbins, "Circuit modeling of the transmissivity of stacked two-dimensional metallic meshes," Opt. Express, vol. 18, no. 13, pp. 13309-133220, Jun. 2010.

[42] R. Marqués, F. Mesa, L. Jelinek, and F. Medina, “Analytical theory of extraordinary transmission through metallic diffraction screens perforated by small holes," Opt. Express, vol. 17, no. 7, p. 5571, Mar. 2009.

[43] J. Carbonell, C. Croenne, F. Garet, E. Lheurette, J. L. Coutaz, and D. Lippens, "Lumped elements circuit of terahertz fishnet-like arrays with composite dispersion," J. Appl. Phys., vol. 108, no. 1, p. 014907, Jul. 2010.

[44] M. Kafesaki, I. Tsiapa, N. Katsarakis, T. Koschny, C. M. Soukoulis, and E. N. Economou, "Left-handed metamaterials: The fishnet structure and its variations," Phys. Rev. B, vol. 75, no. 23, p. 235114, Jun. 2007.

[45] V. Torres, P. Rodríguez-Ulibarri, M. Navarro-Cía, and M. Beruete, "Fishnet metamaterial from an equivalent circuit perspective," Appl. Phys. Lett., vol. 101, no. 24, p. 244101, Dec. 2012.

[46] R. Marqués, L. Jelinek, F. Mesa, and F. Medina, "Analytical theory of wave propagation through stacked fishnet metamaterials," Opt. Express, vol. 17, no. 14, pp. 11582-11593, July 2009.

[47] L. Fu, H. Schweizer, H. Guo, N. Liu, and H. Giessen, "Synthesis of transmission line models for metamaterial slabs at optical frequencies," Phys. Rev. B, vol. 78, no. 11, p. 115110, Sep. 2008.

[48] M. Staffaroni, J. Conway, S. Vedantam, J. Tang, and E. Yablonovitch, "Circuit analysis in metal-optics," Photonics Nanostruct. Fundam. Appl., vol. 10, no. 1, pp. 166-176, 2012.

[49] R. Rodríguez-Berral, F. Mesa, F. Medina, and M. García-Vigueras, "Analytical circuit model for dipole frequency-selective surfaces," in Proc. IMS, Seattle, WA, Jun. 2013.

[50] R. Rodríguez-Berral, F. Mesa, and F. Medina, "Analytical multimodal network approach for 2-D arrays of planar patches/apertures embedded in a layered medium," IEEE Trans. Antennas Propagat., vol. 63, no. 5, pp. 1969-1984, May 2015.

[51] C. Molero, R. Rodríguez-Berral, F. Mesa, F. Medina, and A. B. Yakovlev, "Analytical circuit model for stacked slit gratings," in Proc. IMS, Tampa Bay, FL, Jun. 2014.

[52] R. E. Collin, Field Theory of Guided Waves, 2nd ed. New York: IEEE Press, 1991

[53] S. R. Rengarajan, "Choice of basis functions for accurate characterization of infinite array of microstrip reflectarray elements," IEEE Antennas Wireless Propagat. Lett., vol. 4, pp. 47-50, 2005.
[54] M. Beruete, P. Rodríguez-Ulibarri, V. Pacheco-Peña, M. Navarro-Cía, and A. E. Serebryannikov, "Frozen mode from hybridized extraordinary transmission and Fabry-Pérot resonances," Phys. Rev. B, vol. 87, no. 20, p. 205128, May 2013.

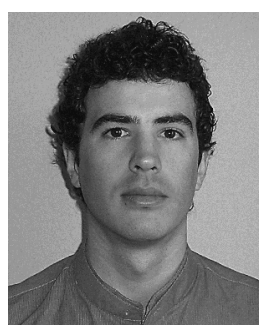

Víctor Torres was born in Pamplona, Spain, in 1985. He received the M.Sci. and Ph.D. degrees in telecommunication engineering from the Public University of Navarre (UPNA), Navarre, Spain, in 2010, and 2014, respectively.

From May 2010 to April 2014, he was working as a Predoctoral Researcher (FPI fellowship recipient) in the Electrical and Electronic Engineering Department, UPNA. He also worked as Visiting Researcher at University of Pennsylvania, Philadelphia, PA, for three months in 2011, at Imperial College London, London, U.K., for three months in 2012 and at the Valencia Nanophotonics Technology Center, Valencia, Spain, for two months in 2012. He is currently working as a Postdoctoral Researcher in the Antenna Group-TERALAB, UPNA. His current research interests are mainly aimed on metamaterials, extraordinary transmission structures, metasurfaces, plasmonics, nanoantennas, biosensing and in general, quasioptical devices at millimeter, terahertz and infrared frequencies.

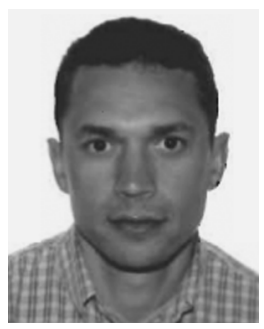

Francisco Mesa (M'93-SM'11-F'14) was born in Cádiz, Spain. He received the Licenciado and Doctor degrees in physics from the Universidad de Sevilla, Seville, Spain.

He is currently Professor in the Departamento de Física Aplicada 1, Universidad de Sevilla, Seville, Spain. His research interests focus on electromagnetic propagation/radiation in planar structures. Since 2001, he has served as an Associate Editor for the IEEE TRANSACtions on MicrowaVe TheOrY \& TECHNIQUES.

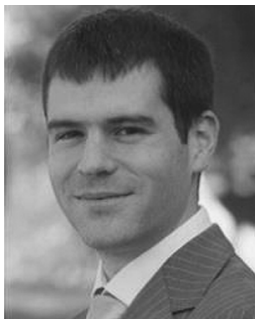

Miguel Navarro-Cía (S'08-M'10-SM'15) was born in Pamplona, Spain, in 1982. He received the M.Sci. and Ph.D. degrees in telecommunication engineering, and the M.Res. degree in Introduction to Research in Communications from Universidad Pública de Navarra, Navarra, Spain, in 2006, 2010, and 2007, respectively.

From September 2006 to January 2010, and from February 2010 until March 2011, he worked as a Predoctoral Researcher (FPI fellowship recipient) and a Research and Teaching Assistant in the Electrical and Electronic Engineering Department, Universidad Pública de Navarra,. He was a Research Associate at Imperial College London and University College London in 2011 and 2012, respectively, and a Junior Research Fellow at Imperial College London from December 2012 until November 2015. Currently, he is Birmingham Fellow in the School of Physics and Astronomy, University of Birmingham. He is also affiliated as a Visiting Researcher with Imperial College London and University College London. He worked as Visiting Researcher at the University of Pennsylvania, Philadelphia, PA, for three months in 2010, at Imperial College London in 2008, 2009 and 2010 for four, six, and three months, respectively, and at Valencia Nanophotonics Technology Center, Valencia, Spain, for two months in 2008. His current research interests are focused on plasmonics, near-field time-domain spectroscopy, metamaterials, antennas, complex surface waves, frequency selective surfaces, and millimeter, terahertz and infrared frequencies.

Dr. Navarro-Cía is a Member of the Optical Society of America, the European Association on Antennas and Propagation (EurAAP), and the Spanish National Association and Professional Board of Telecommunication Engineers. He was awarded the Best Doctoral Thesis in Basic Principles and Technologies of Information and Communications, and Applications corresponding to the XXXI Edition of Awards Telecommunication Engineers 2010, and the 2012 CST University Publication Awards to the best international journal publication using CST Microwave Studio, and was a recipient of the 2011 Junior Research Raj Mittra Travel Grant. 


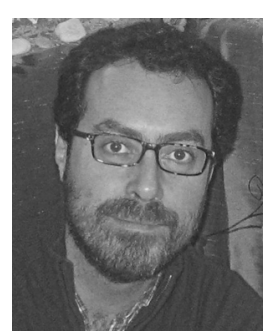

Raúl Rodríguez-Berral was born in Casariche, Seville, Spain, in 1978. He received the M.Sc. (Licenciado) and Ph.D. degrees in physics from the University of Seville, Seville, Spain, in 2001 and 2008, respectively.

In January 2002, he joined the Department of Applied Physics 1, University of Seville, where he is currently an Associate Professor. His research interests include the study of the spectrum and the excitation of periodic and nonperiodic planar structures and high-frequency circuit modeling.

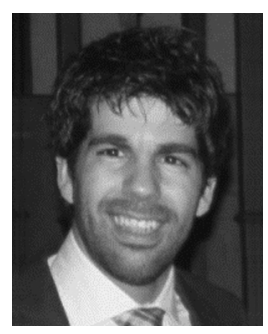

Miguel Beruete was born in Pamplona, Spain, in 1978. He received the M.Sci. and Ph.D. degrees in telecommunication engineering, from the Public University of Navarre (UPNA), Navarre, Spain, in 2002 and 2006, respectively.

From September 2002 to January 2007, he was working as Predoctoral Researcher (FPI fellowship recipient) in the Electrical and Electronic Engineering Department, Public University of Navarre. From January to March 2005 he worked as visiting researcher at the University of Seville, as a part of his doctoral research. From February 2007 to September 2009 he was at the electronics department of the technological center CEMITEC in Noain (Navarre), developing, designing and measuring high frequency communication devices. In September 2009 he joined the TERALAB at UPNA, as a post-doc Ramón y Cajal fellow researcher under the supervision of Prof. Mario Sorolla. In March 2014, he joined the Antennas Group-TERALAB of UPNA, where he supervises several Ph.D. and M.Sci. Theses and is responsible of the TERALAB laboratory. Dr. Beruete has authored more than 100 JCR articles, 3 book chapters, 3 patents and near 250 conference communications. His research interests are directed towards terahertz sensing a communication technology, including metamaterials, plasmonics, extraordinary transmission structures, leaky-wave antennas, nanoantennas, and in general quasioptical devices. His research interests include the study of the spectrum and the excitation of periodic and nonperiodic planar structures and high-frequency circuit modeling.

Dr. Beruete was awarded the Ph.D. Prize from the Public University of Navarre (2006-2007) for the best Doctoral Thesis in the year 2006-2007, two CST University Publication Awards for the best international journal publication using CST in the years 2005 and 2012, and the XII Talgo Award of Technological Innovation in 2011.

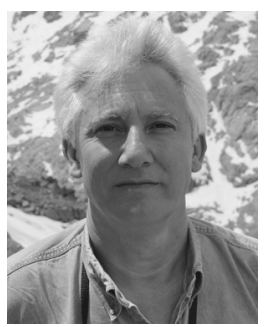

Francisco Medina (M'90-SM'01-F'10) was born in Puerto Real, Cádiz, Spain, in November 1960. He received the Licenciado and Doctor degrees in physics from the University of Seville, Seville, Spain, in 1983 and 1987 respectively.

$\mathrm{He}$ is currently a Professor of Electromagnetism with the Department of Electronics and Electromagnetism, University of Seville, and Head of the Microwaves Group. He has co-authored about 130 journal papers and book chapters and about 280 conference contributions. His research interests include analytical and numerical methods for planar structures, anisotropic materials, and artificial media modeling, including metamaterials. He is in the editorial board of three international journals and acts as Reviewer for more than 40 IEEE, IET, AIP, and IOP journals.

Dr. Medina has been member of the TPCs of several local and international conferences. 